\title{
Impact of Global and Gene-Specific DNA Methylation in de Novo or Relapsed Acute Myeloid Leukemia Patients Treated with Decitabine
}

\author{
Li-Ying Zhang ${ }^{1 \&}$, You-Qing Yuan ${ }^{1 \&}$, Dong-Ming Zhou ${ }^{1}$, Zi-Yan Wang ${ }^{1}$, Song- \\ Guang Ju², Yu Sun ${ }^{1}$, Jun Li ${ }^{1}$, Jin-Xiang Fü ${ }^{1 *}$
}

\begin{abstract}
In this investigation, global DNA methylation patterns and the specific methylation status of 5 genes were studied in DNA from peripheral blood (PB) and impact on progression free survival (PFS) and overall-survival (OS) in patients with de novo or relapsed acute myeloid leukemia (AML) treated with decitabine-based regimens waas assessed. DNA was isolated from PB samples at the time of $\mathbf{- 1 ,}$, and 7 days of chemotherapy. Global methylation was determined by ELISA, and the CpG island DNA methylation profile of 5 genes using a DNA methylation PCR system. Our data demonstrated that patients with a high level of 5-mC had a poor prognosis after demethylation therapy and those who have low levels of 5-mC in PB achieved higher CR and better SO, but there was no significant correlation found between the 5-mC levels and other clinical features before treatment except the disease status. Higher methylation status of Sox 2 and Oct4 genes was associated with differential response to demethylation therapy. A relatively low methylation percentage in one or both of these two genes was also associated with longer OS after decitabine based chemotherapy. We also suggest that global DNA and Oct-4/Sox 2 methylation might impact on the pathogenesis of leukemia and play an important role in the initiation and progression. Moreover, dynamic analysis of 5-mC and Oct-4/Sox 2 in peripheral blood nucleated cells of leukemia patients may provide clues to important molecular diagnostic and prognostic targets.
\end{abstract}

Keywords: Acute myeloid leukemia - global DNA methylation - 5-methylcytosine - Sox2 - Oct4

Asian Pac J Cancer Prev, 17 (1), 431-437

\section{Introduction}

Acute myeloid leukemia (AML) is the most common form of acute leukemia in adults. Clinically, it is characterized by an uncontrolled proliferation and differentiation block in immature hematopoietic stem cells and progenitor cells (HSPCs) resulting in an accumulation of immature blasts (Claus et al., 2010). Despite the presence of a variety of chromosomal aberrations, translocations, and mutations in essential growth and tumor suppressor genes in AML cells, nearly $50 \%$ of patients exhibit a normal karyotype (Riva al., 2012; The Cancer Genome Atlas Research Network. 2013), thus, from a clinical, cytogenetic and molecular stand point, AML is extremely heterogeneous (Dohner et al, 2010; Gu, 2010; Figueroa 2015; Meldi et al., 2015). Although extraordinary progresses in diagnosis and therapy, the outcome of AML patients remains poor, especially in elderly. Less than $50 \%$ of adult AML patients have a 5-year overall survival (OS) whilst about $20 \%$ of elderly survives more than 2 years and the treatment remains a challenge (Dohner et al., 2010; Grossmann et al., 2012; Patel et al.,

\section{2; Kadia et al. 2015).}

Aberrant epigenetic landscapes, including deregulated DNA methylation patterns, are a hallmark of many cancers, including the myeloid malignancies AML. Epigenetic aberrations, including chromatin modifications and changes of DNA methylation have been demonstrated to take up a substantial role during the pathogenesis of AML. Many of the recurrent mutations seen in AML, including those in DNMT3A, IDH1, IDH2, MLL, and EZH2, influence DNA methylation, DNA hydroxymethylation, or histone modification (Ley et al., 2010; Rocquain et al., 2010; Baylin and Jones, 2011; Shih et al., 2012; Kroeze et al., 2014; Cosse et al., 2015). Particularly, DNA hypermethylation at distinct genomic loci is a frequent and specific event during leukemogenesis that is associated with transcriptional gene silencing and that defines subgroups of distinct prognostic and predictive properties (Meldi and Figueroa., 2015; Figueroa et al., 2010). Alterations in DNA methylation, including the hypomethylation of oncogenes and the hypermethylation of tumor suppressor genes, indicate that DNA methylation plays an important role in leukemogenesis. Moreover, 
Noushmehr H and his colleagues (Figueroa et al., 2010) have shown that the global methylation DNA methylation in leukemic blasts is distinct from that seen in normal CD34+ cells, and that DNA methylation patterns alone can segregate AML samples into categories with significant clinical and biological features. Thus, reversal of aberrant DNA methylation events by DNA methyltransferase (DNMT) inhibition represents a biologically rational and clinically promising treatment approach since the decitabine have demonstrated significant single-agent activity in AML (IARC. 2008; Ley et al., 2010; Patel et al., 2012; Wertheim et al., 2012; Meldi et al., 2015). The aim of the present study was to investigate the global DNA methylation pattern and specific methylation status in 5 gene promoters of stem cell related transcription factor Sox2, Oct4, Klf4, C-Myc, and Nanog in DNA from peripheral blood and to correlate these findings with progression free survival (PFS) and overall-survival (OS) after decitabine based chemotherapy in patients with newly diagnosed or relapsed/refractory AML.

\section{Materials and Methods}

\section{Patients}

From Jan 2013 to June 2015, a total of 42 patients with AML were recruited to hematology department of the 2nd hospital of Soochow University, China. Informed consent was obtained from every patient for laboratory studies according to the guidelines of Committee of Medical Ethics of Soochow University Hospitals. The median age was 63(20-78) years. According to FrenchAmerican-British (FAB) classification system, there were M0 (n=3), M1 ( $n=6)$, M2 ( $=19)$, M3 ( $n=1)$, M4 ( $=6)$ and M5 $(n=7)$. Main characteristics of the patients are shown in Table 1. BM aspirates were harvested directly after formal written consent was obtained. Bone marrow mononuclear cells (MNCs) were separated by FicollHypaque density gradient centrifugation. The metaphase chromosomes were banded by the R-banding techniques and karyotype according to the International System for Human Cytogenetic Nomenclature (Tien et al., 1995). A panel of monoclonal antibodies to myeloid-associated antigens, including CD13, CD33, CD11b, CD15, CD14, and CD41a, as well as lymphoid-associated antigens, including CD2, CD5, CD7, CD19, CD10, and CD20, and lineage nonspecific antigens HLA-DR, CD34, and CD56, were used to characterize the immunophenotypes of the leukemia cells.

All patients were divided into three groups according to their disease status. The groups were as fellows: 1) newly diagnosed AML patients $(n=20) ; 2)$ relapsed/ refractory AML patients (n-10); and 3) AML patients who had already got completed remission $(n=12)$ as positive controls(only for consoliation therapy). All included patients were receiving the same treatment protocol, but the elder(age $\geq 65$ ) patients received relatively low dose chemotherpy ; in brief, they received 1-2 cycles of decitabine based induction chemotherapy (decitabine $20 \mathrm{mg} / \mathrm{m}^{2} \times 3-5$ days, aclacinomycin $10 \mathrm{mg} / \mathrm{m}^{2} \times 5$ days, Ara-C $10 \mathrm{mg} / \mathrm{m}^{2} \mathrm{q} 12 \mathrm{~h} \times 10-14$ days, and G-CSF $150 \mu \mathrm{g} /$ $\mathrm{d} \times 10-14$ days) depending upon BM aspiration done at the end of each induction course (post-induction remission). Additional consolidation regimens included 1 cycles of DA $\times 7$ days (doxorubicin $45-60 \mathrm{mg} / \mathrm{m}^{2}+$ Ara-C $\left.100 \mathrm{mg} / \mathrm{m}^{2}, \mathrm{q} 12 \mathrm{~h}\right)$. Then, patients received intermittent chemotherapy(Ara-C 1.5-2.5g, q12h×3 days) every 2 months for 4- 6 cycles with the standard follow-up care and regular BM aspiration to confirm remission. The patients undergo follow up.

\section{DNA isolation}

Blood samples from all AML patients were obtained at $-1,1$, and 7 pre- and post-chemotherapy. Mononuclear cells (MNCs) were separated by Ficoll-Hypaque density gradient centrifugation. Genomic DNA was isolated from NMCs. Briefly, The extraction was performed using the commercial DNeasy Blood \& Tissue Kit (Qiagen, Hilden, Germany) according to manufacturer's protocol and the 260/280 nm absorbance ratio was used to assess the purity of DNA.

\section{Global and specific DNA methylation analysis}

Global methylation status was determined in all patients by a specific ELISA assay (MethylFlashTM Methylated DNA quantificacion Kit; Epigentek, Farmingdale, NY, USA) in total DNA, which is based on recognition and quantification of the DNA methylated fraction by a 5-methylcytosine (5-mC) antibody. The assay was performed according to manufacturer's protocol.

\section{Specific-gene DNA methylation analysis}

Specific-gene DNA methylation was determined in $\mathrm{CpG}$ islands of 5 genes in 42 patients with enough DNA by a DNA methylation PCR system based on DNA digestion with methylation sensitive and/or dependent restriction enzymes (Methyl-ProfilerTM DNA Methylation PCR Array System; Qiagen, Hilden, Germany). According to manufacturer's protocol, DNA was treated with sensitive and/or dependent restriction enzymes, active against methylated and unmethylated DNA, respectively. The genes selected and studied shown in Table 2.

\section{Data analysis}

Statistical analyses of data were performed using the Statistical Package for the Social Sciences (SPSS) software version 17.0 (SPSS Inc., Chicago, IL, USA) and R 2.9.2 soft-ware (Vienna, Austria, 2008). KolmogorovSmirnov test was done to test the normality of data distribution. All p values were two-tailed and the threshold of statistical significance was p less than 0.05. Chi square test was used to compare groups. Quantitative data were presented as median, range, mean and standard deviation. For comparison between two groups; student t-test and Mann-Whitney test (for non-parametric data) were used. For comparison between more than two groups; ANOVA and Kruskal-Wallis (for non-parametric data) were used. Clinical and biological parameters were recorded at the time of diagnosis. Descriptive statistical analysis of the main characteristics of patients was performed. Association of global and specific DNA methylation status with PFS and OS, was determined using the Kaplan-Meier method and log-rank test. The cut-off value to dichotomize 
Global and Gene-specific DNA Methylation in de Novo or Relapsed AML Patients Treated with Decitabine

the continuous variables associated with PFS or OS was calculated by Graphpad Prism 5.0 software.

\section{Results}

All 30 patients were evaluable for response. Overall response (OR) was achieved in $73.4 \%$ of the patients (complete remission(CR) 60\%, partial response(PR) $13.4 \%$ and no response $26.6 \%$ ) after finishing the 2 nd circle of induction therapy. Among 20 newly diagnosed AML patients total of 15 patients got CR and $2 \mathrm{PR}$, while only 3 relapsed/refractory AML patients got CR and two PR. The median PFS and OS after decitabine based therapy were 11.5 and 25.6 months, respectively. Our data demonstrated that there was a trend to a longer OS and PFS in the case of low global methylation status and specific gene methylation status after treatment.
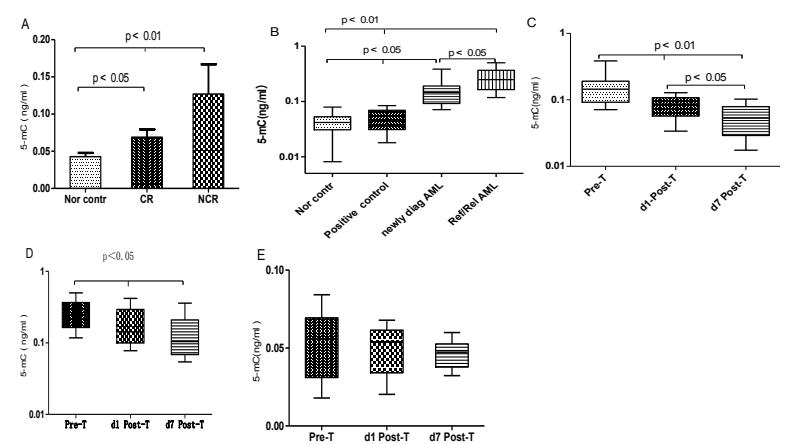

Figure 1. Changes of Methylation Status in Patients with AML before and after Decitabine Based Chemotherapy. A) The levels of 5-mC in patients among the different groups after demethylation treatment; B) The changes of 5-mC concentraions in different AML groups; C) The dynamic changes of 5-mC concentrations in new diagnosed AML patients before and after demethylation therapy; D) The dynamic changes of 5-mC concentration in refractory/relapsed AML patients before and after demethylation therapy; D) The concentrations of 5-mC in AML patients who got CR

\section{Global methylation status}

A low global methylation status was observed in these who had already got CR and normal controls group $(0.043 \pm 0.018 \mathrm{ng} / \mathrm{ml}$ and $0.052 \pm 0.089 \mathrm{ng} / \mathrm{ml}$ respectively). A high level of 5-mC was detected among the de novo AML and relapsed/refractory AML patients. The value was $0.16 \pm 0.089 \mathrm{ng} / \mathrm{ml}$ and $0.136 \pm 0.043 \mathrm{ng} / \mathrm{ml}$ respectively. The level of 5-mC of newly diagnosed or relapsed/refractory AML was higher than those in normal and positive controls $(\mathrm{p}<0.05)$. A decrease of global methylation status was observed in all the patients treated with decitabine based chemotherapy at day 1 or days 7 , but it seemed that the 5-mC levels decreased significantly at day 7 in the de novo AML and relapsed/refractory AML groups, there was statistical significant between the newly diagnosed AML (day1, 0.0838 $\pm 0.030 \mathrm{ng} / \mathrm{ml}$ and day7, $0.055 \pm 0.028 \mathrm{ng} / \mathrm{ml}$ ) and relapsed/ refractory AML patient (day1,0.248 $\pm 0.142 \mathrm{ng} / \mathrm{ml}$ and day7, $0.155 \pm 0.103 \mathrm{ng} / \mathrm{ml}$ ) as in comparison with normal controls or positive controls $(\mathrm{p}<0.05)$ (Figure 1a-d). Our data showed that there were no significance of the 5-mC levels of among with the age, gender, FAB classification, white blood cell count, blasts, hemoglobin, and platelet before treatment except the disease status (Table 1).

The value obtained at day 7 by MaxStat to dichotomize the patient group was $0.113 \mathrm{ng} / \mathrm{ml}$ and there were 17 patients whose $5-\mathrm{mC}$ level higher than $0.113 \mathrm{ng} / \mathrm{ml}$ and the others were less than $0.113 \mathrm{ng} / \mathrm{ml}$. Patients with less than $0.113 \mathrm{ng} / \mathrm{ml}$ of total DNA methylated achieved higher CR than patients with more methylated DNA and also those patients achieved better OS and PFS (median 21.3 versus 15 months $)(p=0.004$; Figure 1$)$

\section{Specific-gene methylation status}

Concerning methylation on specific-genes, except to Nanog, the methylation status in pre-treatment patients tended to increase according to the disease status. Our data showed that only a low status of partially methylated Sox 2 and Nanog detected among the normal controls

Table 1. Demographic and Laboratory Data of the Studied Patients

\begin{tabular}{|c|c|c|c|}
\hline Variable & $\mathrm{n}$ & 5-Mc(range) & $\mathrm{p}$ \\
\hline Median age, year(range) & $63(20-78)$ & $0.112(0.052-0.501)$ & 0.56 \\
\hline Gender(M/F) & $22 / 20$ & $0.143(0.052-0.470)$ & 0.18 \\
\hline \multicolumn{4}{|l|}{ Disease status } \\
\hline De novo AML & 20 & $0.16(0.071-0.385)$ & 0.034 \\
\hline RR AML & 10 & $0.27(0.18-0.501)$ & 0.027 \\
\hline $\mathrm{CR}$ & 12 & $0.052(0.018-0.084)$ & 0.72 \\
\hline \multicolumn{4}{|l|}{ FAB subtypes } \\
\hline M0 & 3 & $0.098(0.074 \sim 0.158)$ & \multirow[t]{6}{*}{0.73} \\
\hline M1 & 6 & $0.120(0.020 \sim 0.30)$ & \\
\hline M2 & 19 & $0.114(0.020 \sim 0.501)$ & \\
\hline M3 & 1 & 0.05 & \\
\hline M4 & 6 & $0.113(0.071 \sim 0.158)$ & \\
\hline M5 & 7 & $0.152(0.076 \sim 0.385)$ & \\
\hline \multicolumn{2}{|c|}{ Immunophenotype(myeloid/mixed) } & $40 / 2$ & 0.076 \\
\hline \multicolumn{2}{|c|}{ Cytogenetic(normal/abnormal) } & $19 / 23$ & 0.055 \\
\hline \multicolumn{2}{|c|}{ Blasts in BM, \%(range) } & $30.5(1.5 \sim 88)$ & 0.08 \\
\hline \multicolumn{2}{|c|}{$\mathrm{WBC} \times 10^{9} / \mathrm{L}$, median(range) } & $4.5(2.5 \sim 134.8)$ & 0.15 \\
\hline \multicolumn{2}{|c|}{$\mathrm{Hb}(\mathrm{g} / \mathrm{L})$, median(range) } & $84(48 \sim 133)$ & 0.72 \\
\hline \multicolumn{2}{|l|}{ Plt $\times 10^{9} / \mathrm{L}$, median(range) } & $55(3 \sim 320)$ & 0.09 \\
\hline
\end{tabular}




\begin{tabular}{|c|c|c|c|c|c|}
\hline Genes & primers & Primers 5' -3' & $\begin{array}{c}\text { Annealing temperature } \\
(\mathrm{oC}) / \text { cycles }\end{array}$ & $\begin{array}{l}\text { Production } \\
\text { length (bp) }\end{array}$ & $\begin{array}{c}\text { Accession } \\
\text { number }\end{array}$ \\
\hline \multirow{2}{*}{ Sox 2} & M-MSP & $\begin{array}{c}\text { F:TTAATAAGAGAGTGGAAGGAAATTTAGA } \\
\text { R:AAATAAAAACTCAAAAATCTTACCCG }\end{array}$ & $55 / 35$ & 214 & \\
\hline & U-MSP & $\begin{array}{c}\text { F:TAATAAGAGAGTGGAAGGAAATTTAGAT } \\
\text { R: ATAAAAACTCAAAAATCTTACCCACC }\end{array}$ & $55 / 35$ & 212 & \\
\hline \multirow{2}{*}{ 4-Oct } & M-MSP & $\begin{array}{l}\text { F: ATAAAAATAGGAAGGAGTTTTTCGC } \\
\text { R: CTATCAAACAAATACAACAACGTCG }\end{array}$ & $55 / 35$ & 200 & \\
\hline & U-MSP & $\begin{array}{l}\text { F: AAAAATAGGAAGGAGTTTTTTGTGA } \\
\text { R: ATCAAACAAATACAACAACATCAAC }\end{array}$ & $53 / 35$ & 196 & \\
\hline \multirow{2}{*}{ Klf4 } & M-MSP & $\begin{array}{c}\text { F: TTTATTTTTTAGTAGAAGGGTTCGC } \\
\text { R: ATAAAATCAACGAACGACACGAT }\end{array}$ & $57 / 35$ & 242 & \\
\hline & U-MSP & $\begin{array}{l}\text { F: TATTTTTTAGTAGAAGGGTTTGTGA } \\
\text { R: TAATAAAATCAACAAACAACACAAT }\end{array}$ & $55 / 35$ & 242 & \\
\hline \multirow{2}{*}{ C-Myc } & M-MSP & $\begin{array}{l}\text { F: TCGTTGGAAATTTTGTATTTCG } \\
\text { R: CCCTCTCTAAACAACACCCG }\end{array}$ & $55 / 37$ & 152 & \\
\hline & U-MSP & $\begin{array}{l}\text { F: TTGTTGGAAATTTTGTATTTTGG } \\
\text { R: CCCTCTCTAAACAACACCCAC }\end{array}$ & $55 / 37$ & 152 & \\
\hline \multirow{2}{*}{ Nanog } & M-MSP & $\begin{array}{l}\text { F: GTTGTTTAGGTTGGAGTATAGTGGC } \\
\text { R: AAATTAAAAAACCGTAACAAACGAA }\end{array}$ & $57 / 35$ & 229 & \\
\hline & U-MSP & $\begin{array}{l}\text { F: GTTGTTTAGGTTGGAGTATAGTGGTG } \\
\text { R: AAATTAAAAAACCATAACAAACAAA }\end{array}$ & $55 / 35$ & 229 & \\
\hline
\end{tabular}

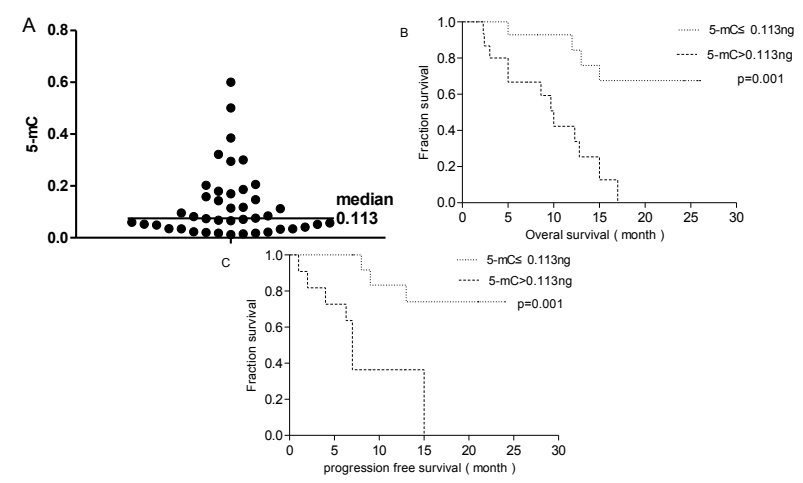

Figure 2. The Distribution of 5-mC Levels at Day 7 after Decitabine Based Chemotherayp and its Impaction on OS and PFS

(15.4\% and $30 \%$ respectively), while Oct4, C-Myc, and Klf4 genes methylation were not detected. A relatively low methylation percentage of 5 specific-genes methylation status was detected in patients who had already got CR. The ratio of Sox2, Oct4, Klf4, C-myc and Nanog gene methylation was $50 \%, 50 \%, 41.7 \%, 33.3 \%$, and $100 \%$ respectively. The highest percentage of methylation status associated with those newly diagnosed or relapsed/ refractory AML patients. they were 90\%, 76.7\%, 70\%, $86.7 \%$ and $100 \%$ respectively and was higher than those in CR patients ( $\mathrm{p}<0.05$; Figure 3 ). The methylation status decreased after demethylation therapy. Statistic analysis with methylation status for all these genes showed that Sox 2 and Oct 4 gene associated with a differential profile to demethylation therapy, showing the responders a lower methylation status ( $p=0.029$, Figure 3 ). A relatively low methylation percentage in these two genes or both was also associated with longer OS after decitabine based

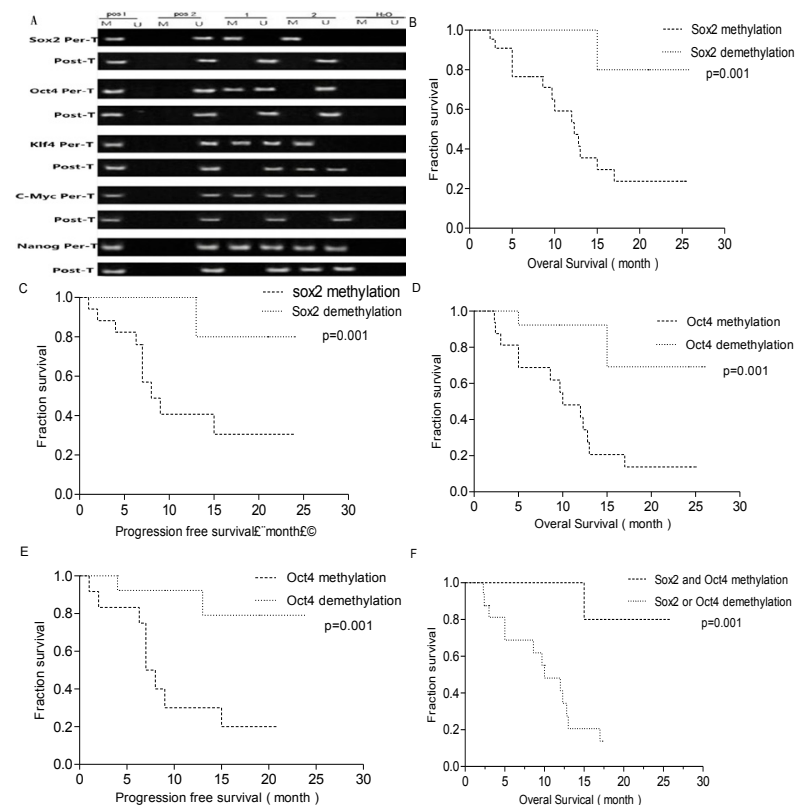

Figure 3. The Impact of Specific Gene Methylation on OS and PFS of Patient with AML after Decitabine Based Chemotherayp. Our resutlts showed that a higher Sox2 or/and Oct4 methylation status identified a specific subset of patients with an extremely short OS and PFS(B-F). For patients with none, one or two factors, the OS was 17.6, 11.7 and 6.3 months respectively

chemotherapy $(\mathrm{p}=0.015$; Figure 4$)$.

The combination of two factors (1) a relatively high methylated global genome ( $>0.113)$ and (2) a higher Sox2 or/and Oct 4 methylation status identified a specific subset of patients with an extremely short OS. This combination of both factors allowed the identification of three subsets 
of patients with different median OS. For patients with none, one or two factors, the OS was $17.6,11.7$ and 6.3 months respectively $(\mathrm{p}=0.005)$.

No other factors were associated with a different OS at the univariate analysis, except the blasts in bone marrow. At multivariate analysis including age, FAB classification, gender and white blood cell count, the variables previously established as prognostic in OS such as gene mutation, global DNA methylation and specific gene methylation status, were the only ones retaining statistical significance (Table 1). Concerning PFS, the number of previous treatment lines had significant prognostic impact which was retained at multivariate analysis. There was also a trend to a shorter PFS in the case of Sox 2 or Oct 4 methylation status whether in novo or relapsed AML.

\section{Discussion}

Aberrant regulation of DNA methylation is characteristic of cancer cells and clearly influences phenotypes of various malignancies. Although AML has been traditionally thought of as a genetic disease driven by the somatic acquisition of multiple oncogenic mutations, recent studies have clearly shown that it is also an epigenetic disease and that dysregulation of DNA methylation and/or chromatin structure plays a central role in leukemogensis (Corces-Zimmerman et al., 2014; Kadia et al., 2015). Despite these associations, routine multilocus assessment of epigenetic phenomena is not common, due in part to the difficulty and expense involved in establishing and performing epigenetic assays. We found that a relatively high DNA global methylation was an independent prognostic factor in patients AML before treatment and those whose methylation status decreased after demethylation therapy seemed to have higher CR rate and longer PFS. Of note, these results were obtained by Elisa, a cheap and fast technique that should be clinically explored in prospective studies.

Claus R and colleagues (Claus et al., 2010; Pollyea et al., 2011) demonstrated that high DNA methylation in the AML patients before treatment and found decitabineinduced DNA methylation changes occur very early and imply mechanisms distinct from non-hypomethylating cytosine analogs. Our results demonstrate the feasibility of monitoring early DNA methylation changes in AML patients undergoing treatment with DNA methyltransferase (DNMT) inhibitors, at a time when results are not potentially confounded by selection of resistant cells or even replacement with normal hematopoietic cells. Pretreatment methylation patterns demonstrated consistently elevated DNA methylation in the AML patient samples compared to healthy controls. Following decitabine based treatment, both global and genes specific hypomethylation events were detectable as early as one days after exposure which indicates that epigenetic changes are induced early after the start of therapy and hypomethylation status showed a time depend manner. Furthermore, distinct pretreatment DNA methylation differences and the course of AML pathogenesis might explain heterogeneity of demethylation patterns. Cells from patients with relapsed/ refractory AML, for example, were shown to exhibit more extensive aberrant DNA methylation and higher 5-mC. Our data also demonstrated that the dynamically detecting the change of 5-mC levels during the decitabine-based therapy might be useful as potential tumor burden in the prognosis of patients with AML.

Uncontrolled self-renewal is proposed to be an important mechanism in carcinogenesis and on the basis of the cancer stem cell (CSC) hypothesis, a tumor may be sustained by a subset of cancer cells with stem cell-like features that have the ability for self-renewal and pluripotency (Mazzarella et al., 2014). It was first discovered in 2006 by Takahashi and Yamanaka through the overexpression of four key transcription factors, Oct4, Sox 2, Klf4 and c-Myc, which resultes changes in the epigenome of the cells, therefore the cells requires and re-establishs the characteristic of pluripotent stem cell (Firas et al., 2015; Takahashi and Yamanaka, 2006). Recent studies(Claus et al., 2013; Bullinger et al., 2010; Kon Kin et al., 2015) were confirmed that the Oct4Sox2-Nanog network was required for the activation of self-renewal regulators and showed that several genes have been identified as hypermethylated, such as tumor suppressor genes, cell-cell signaling and cell adhesion molecules. Thus, hypermethylation of genes might involve in myelopoiesis. But how about the methylation changed on self-renewal genes during the myelopoiesis was not clinically explored. Particularly, DNA hypermethylation at distinct genomic loci is a frequent and specific event during leukemogenesis that is associated with transcriptional gene silencing and that defines subgroups of distinct prognostic and predictive properties (Zhao et al., 2015). Amin S and colleagues (Amini et al., 2014) had demonstrated that stem cell markers Oct4, Nanog, Sox2, nucleostemin, Bmi, Zfx, Esrrb, Tcl1, Tbx3, and Dppa4 significantly express in cancer cell lines and cancer tissues. Hence, these markers might be useful as potential tumor markers in the diagnosis and/or prognosis of tumors.

By using methylation- specific PCR, we reports for the first time the methylation status of self-renewal regulator factors such as Oct4, Sox2, C-myc, Klf-4 and Nanog pre- and post decitabine-based chemotherapy. These genes are required for efficient self-renewal of normal hematopoietic stem cells and/or leukemia stem cells. Our results demonstrated that the methylation status of those genes tended to increase before treatment except the Nanog. A low status of partially methylated Sox 2 and Nanog status was found among the normal controls $(15.4 \%$ and $30 \%$ respectively), while methylation of Oct 4 , C-Myc and Klf4 gene was not detected. The highest percentage of methylation status of Oct4, Sox2, C-myc, and Klf-4 was associated with those novo or relapsed/ refractory AML patients, which were 90\%, 76.7\%, 70\%, and $86.7 \%$ respectively and was higher than those in CR patients $(\mathrm{p}<0.05)$. Zhao et al. (2015) found a significant correlation between the expression of OCT-4 and the proportion of peripheral blood naïve cells, which serve as an important parameter for leukemia diagnosis and prognosis. Interestingly, our results showed methylation status of Sox 2 and Oct4 gene associated with a differential profile to demethylation therapy, showing the responders a lower methylation status ( $\mathrm{p}=0.029$, Figure 3 ). A relatively 
low methylation percentage in these two genes or both was also associated with longer OS after decitabine based chemotherapy $(p=0.015 ;$ Fig. 3$)$. Interestingly, our results showed no correlation among the methylation of those two genes with FAB classification, white blood cell count, hemoglobin, and platelet before treatment except the disease status (data no showed), implying that the OCT-4/ Sox 2 mainly presented on the leukemia stem/progenitor cells. These data seem consistent with the recent finding that Oct-4 maintains non small cell lung cancer cells in a poorly differentiated state through a mechanism that depends on promoting cell proliferation. The observation is also consistent with the previously published data showing the role of OCT-4 in other solid tumors, such as in malignant ovarian tumors reported by Peng (Peng et al., 2010; Chen et al., 2012) and in breast cancer reported by Liu et al. (2102).

In conclusion, our study demonstrated that there is a high level of global DNA methylation status in peripheral blood(PB) MNCs of active leukemia patients. The patients with high level of 5-mC has a poor prognosis after demethylation therapy and those who have low levels of $5-\mathrm{mC}$ in $\mathrm{PB}$ achieved higher CR and better OS, but there is no significant correlation found among the 5-mC levels of with the age, gender, FAB classification, white blood cell count, blasts, hemoglobin, and platelet before treatment except the disease status. Our data also showed that higher percentage of methylation of Sox 2, Oct4, Klf4, and C-myc gene found among the patients of newly diagnosed or refractory/relapsed AML. Higher methylation status of Sox 2 and Oct 4 gene associated with a differential profile to demethylation therapy. A relatively low methylation percentage in these two genes or both was also associated with longer OS after decitabine based chemotherapy. This observation is of considerable significance since global DNA methylation and gene-specific DNA methylation is known to have an oncogenic activity. We also suggest that global DNA methylaiton and Oct-4/Sox 2 might impact in the pathogenesis of leukemia and play an important role in the initiation and progression of leukemia. Moreover, the dynamic analysis the 5-mC and Oct-4/Sox 2 in peripheral blood nucleated cells of leukemia patients may serve as an important molecular diagnostic and prognostic target in leukemia.

\section{References}

A Language and Environment for Statistical Computing. In: Computing RFfS, editor. Vienna Austria; 2008. http:// www.R-project.org.

Amini S, Fathi F, Mobalegi J, et al (2014). The expressions of stem cell markers: Oct4, Nanog, Sox2, nucleostemin, Bmi, Zfx, Tcl1, Tbx3, Dppa4, and Esrrb in bladder, colon, and prostate cancer, and certain cancer cell lines. Anatomy Cell Biol, 47, 1-11.

Baylin SB, Jones PA (2011). A decade of exploring the cancer epigenome - biological and translational implications. Nat Rev Cancer, 11, 726-34.

Bullinger L, Ehrich M, Doner K, et al (2010). Quantitative DNA methylation predicts survival in adult acute myeloid leukemia. Blood, 115, 636-42.

Claus R, Plass C, Armstrong SA, et al (2010). DNA methylation profiling in acute myeloid leukemia: from recent technological advances to biological and clinical insights. Future Oncol, 6, 1415-31.

Claus R, Pfeifer D, Almstedt M, et al (2013) Decitabine induces very early in vivo DNA methylation changes in blasts from patients with acute myeloid leukemia. Leukemia Res, 37, 190-6.

Chen Z, Wang T, Cai L, et al (2102). Clinicopathological significance of non-small cell lung cancer with high prevalence of Oct-4 tumor cells, J Exp Clin Cancer Res, 31, 10.

Corces-Zimmerman MR, Hong WJ, Weissman IL, et al (2014). Preleukemic mutations in human acute myeloid leukemia affect epigenetic regulators and persist in remission. PNAS 111, 2548-53.

Cosse G, Celton M, Lamontagne V, et al (2015). Whole genome and transcriptome analysis of a novel AML cell line with a normal karyotype. Leuk Res, 39, 709-18.

Dohner H, Estey EH, Amadori S, et al (2010). Diagnosis and management of acute myeloid leukemia in adults: recommendations from an international expert panel, on be half of the European Leukemia Net. Blood, 115, 453-474.

The Cancer Genome Atlas Research Network (2013). Genomic and epigenomic landscapes of adult de novo acute myeloid leukemia. N Engl J Med, 368, 2059-2074.

Figueroa ME, Lugthart S, Li Y, et al (2010). DNA methylation signatures identify biologically distinct subtypes in acute myeloid leukemia. Cancer Cell, 17, 13-27.

Firas J, Liu X, Lim SM, et al (2015). Transcription factormediated reprogramming: epigenetics and therapeutic potential. Immunol Cell Biol, 1-6.

Grossmann V, Schnittger S, Kohlmann A, et al (2012). A novel hierarchical prognostic model of AML solely based on molecular mutations. Blood, 120, 2963-72.

Gu H, Bock C, Mikkelsen TS, et al (2010). Genomescale DNA methylation mapping of clinical samples at single-nucleotide resolution. Nature Methods, 7, 133-6.

Kadia TM, Ravandi F, O'Brien S, et al (2015). Progress in acute myeloid leukemia. Clinical Lymphoma, Myeloma Leukemial, 5, 139-51.

Kon Kin T, Gore SD, Zeidan AM (2015). Epigenetic therapy in acute myeloid leukemia: Current and future directions; Semin Hematol, 52, 172-82.

Kroeze LI, Aslanyan MG, van Rooij A, et al (2014). Characterization of acute myeloid leukemia based on levels of global hydroxymethylation. Blood, 124, 1110-8.

Ley TJ, Ding L, Walter MJ, et al (2010). DNMT3A mutations in acute myeloid leukemia. $N$ Engl J Med, 363, 2424-33.

Liu C, Cao X, Zhang Y, et al (2012). Co-expression of Oct-4 and Nestin in human breast cancers. Mol Biol Rep, 39, 5875-81.

Lyon (2008). International Agency for Research on Cancer (IARC), 110-147

Mazzarella L, Riva L, Luzi L, et al (2014) The Genomic and Epigenomic Landscapes of AML. Semin Hematol, 51, 259-72.

Meldi KM, Figueroa ME (2015). Cytosine modifications in myeloid malignancies. Pharmacol Therapeutics.

Noushmehr H, Weisenberger DJ, Diefes K, et al (2010). Cancer genome atlas research network: identification of a $\mathrm{CpG}$ island methylator phenotype that defines a distinct subgroup of glioma. Cancer Cell, 17, 510-22.

Patel JP, Gonen M, Figueroa ME, et al (2012). Prognostic relevance of integrated genetic profiling in acute myeloid leukemia. N Eng J Med, 366, 1079-89.

Meldi K, Qin T, BuchiF, et al (2015). Specific molecular signatures predict decitabine response in chronic myelomonocytic leukemia. J Natl Cancer Inst, 125, 1857-72. 
Peng S, Maihle NJ, Huang Y (2010). Pluripotency factors Lin28 and Oct4 identify a subpopulation of stem cell-like cells in ovarian cancer. Oncogene. 29, 2153-9.

Pollyea DA, Kohrt HE, Gallegos L, et al (2011). Safety, efficacy and biological predictors of response to sequential azacitidine and lenalidomide for elderly patients with acute myeloid leukemia. Leukemia, 26, 893-901.

Riva L, Luzi L, Pelicci PG (2010) Genomics of acute myeloid leukemia: the next generation. Front Oncol, 2, 40.

Rocquain J, Carbuccia N, Trouplin V, et al (2010). Combined mutations of ASXL1, CBL, FLT3, IDH1, IDH2, JAK2, KRAS, NPM1, NRAS, RUNX1, TET2 and WT1 genes in myelodysplastic syndromes and acute myeloid leukemias. BMC Cancer, 10, 401-13.

Shih AH, Abdel-Wahab O, Patel JP, et al (2012). The role of mutations in epigenetic regulators in myeloid malignancies. Nat Rev Cancer, 12, 599-612.

Takahashi K, Yamanaka S (2006). Induction of pluripotent stem cells from mouse embryonic and adult fibroblast cultures by defined factors. Cell, 126, 663-76.

Tien HF, Wang CH, Lin MT, et al (1995). Correlation of cytogenetic results with immunophenotype, genotype, clinical features, and ras mutation in acute myeloid leukemia: a study of 235 Chinese patients in Taiwan. Cancer Genet Cytogenet, 84, 60-8.

Wertheim GB, Hexner E, Bagg A (2012). Molecular-based classification of acute myeloid leukemia and its role in directing rational therapy: personalized medicine for profoundly promiscuous proliferations. Mol Diagn Ther, 16, 357-69.

Zhao Q, Ren H, Feng S, et al (2015). Aberrant expression and significance of OCT-4A transcription factor in leukemia cells. Blood Cells Molecules Diseases, 54, 90-6. 